\title{
Treatment of condensation in sandwich panels without known vapour resistance of sealant
}

\author{
Gregor Vidmar * \\ Slovenian national Building and Civil Engineering Institute, 1000 Ljubljana, Slovenia
}

\begin{abstract}
In order to calculate amount of interstitial condensation in a building envelope, water vapour resistance of each layer is of importance. Once having it, 1D calculation according to ISO 13788 with monthly average vapour pressures can be applied. In EN 14509 sandwich panels are considered to be impermeable for water vapour, thus (according to the standard) water vapour cannot enter from outside and condensate in the panels. But it is not always true for real sandwich panels, because joints between neighbouring panels can cause non-neglecting water vapour bridges. Although in measurements of linear water vapour transmittance of the joints $\left(\Psi_{v}\right)$ stationary boundary vapour pressures can be applied, the measurements can be long lasting. We shortened time needed to get $\Psi_{v}$ performing simulations in Delphin 6.0. We simulated panels and steel sheets with joints using constant boundary vapour pressures and compared the results with the results of measurements on the equivalent systems.

In systems under consideration a sealant in built-in-state, located at a joint of a sandwich panel, is a compressed EPDM tube. It is impossible to directly measure its effective $\mu$ according to ISO 12572. In the paper we study to which precision it is possible to determine it using measurements and simulations. Once having effective $\mu$ of the sealant (if all other necessary material parameters available) one can simulate condensation in envelopes including sandwich panels in 2D according to EN 15026 using hourly climatic data. Another goal of the study was determination of differences in resulting $\Psi_{v}$ values when varying narrowest part of the gap $d_{G A P}$ at the joint in the panels without any sealant. Results confirm significant sensibility of $\Psi_{v}$ to variations of $d_{G A P}$.
\end{abstract}

\section{Introduction}

Significant proportion of nowadays industrial buildings is built from sandwich panels, which are composed from two steel sheets and thermal insulation in-between. As for every building element, it is of great importance to check, whether water vapour can condense at the surface of sandwich panels or even in the panels.

Surface condensation can be treated according to ISO 13788 or DIN 4108-3, when using surface temperature calculation according to ISO 6946 or ISO 10211. Surface condensation at inner surface in the neighbourhood of the joint of a cassette facade element, was numerically studied in [1] (cassette elements usually have larger thermal bridge than sandwich panels). In the system relative humidity at inner surface was about $20 \%$ higher in the neighbourhood of the joint than far away from it. Therefore condensation near the joint is by far more probable than at the centre of the panel. With additional thermal insulation of the whole panel system at outer side, the possibility for water vapour condensation at inner surface was removed.

Water vapour condensation in a construction from sandwich panels attached to cassette panels was studied in [2]. In the study sandwich panel is treated as fully vapour impermeable, whereas joints between two cassette elements are treated with slots, because their metal parts run across entire element, which is usually not the case in sandwich panels. Under considered boundary conditions calculated relative humidity in the system was low enough, that no condensation appeared. Also, according to EN 14509 sandwich panels are treated as fully closed for water vapour. Namely, according to EN ISO 10456 and EN ISO 13788 water vapour diffusion resistance $\mu$ of the steel sheets is taken as infinity, while if the value is needed for computational purposes it can be "diminished" to $10^{6}$ (ISO 10456) or $10^{5}$ (ISO 13788). In some software tools for highly vapour resistant materials "vapour tight" flag can be marked and real (high) $\mu$ values is not needed indeed.

But sandwich panels also have the joints between neighbouring panels; therefore, in reality they are never fully impermeable for air or water vapour. If their water vapour resistance is necessary to be quantified, one has to take into account linear water vapour bridges. From linear water vapour bridges linear water vapour transmittance $\psi_{\mathrm{v}}$ is determined and can be measured or simulated in similar way as linear thermal transmittance $\psi$ according to EN ISO 10211 or EN ISO 14683.

An experimental study of linear water vapour transmittance of several joints of sandwich panels is presented in [3]. Three different configurations were studied: 1.) configuration without any sealant between two panels, 2.) configuration with a sealant at the inner part and no sealant at the outer part of the panel's joint and 3.) configuration with two sealants, one at the inner

\footnotetext{
* Corresponding author: gregor.vidmar@zag.si.
} 
part and one at the outer part of the panel's joint. Comparison between panel system without a joint and systems with joints was not done in the study from [3], unfortunately, because a system without joints was not measured. The water vapour flux through the specimen was measured with stationary boundary conditions using guarded water vapour chambers imitating guarded hot plate method from the thermal conductivity measurement according to EN 12667. In order to shorten the time needed to obtain stationary results a transient fitting procedure with 2 fitting parameters was invented. Such experiments with the existed equipment cannot be performed in a certain random chosen real climatic circumstance. Therefore, numerical simulations can be chosen.

Water vapour characteristic of the sealant is one of the crucial factors one needs to perform valuable numerical simulations and consequently find out, how a panel behaves due to possibility of interstitial vapour condensation. Due the shape of the compressed sealant and consequent impossibility of measuring effective water vapour resistance sealant directly according to ISO 12572 (sealant does not have parallel surfaces and due to the air hole it is not homogenous), we present here an attempt to estimate the precision, with which it is possible to determine it using results of few long lasting experiments from [3] and a couple of numerical simulations in Delphin 6.0. Additionally, we study the sensibility of the final vapour flux to the narrowest gap width.

\section{Methodology}

Schematic picture of a joint in a sandwich panel is presented in Fig. 1.

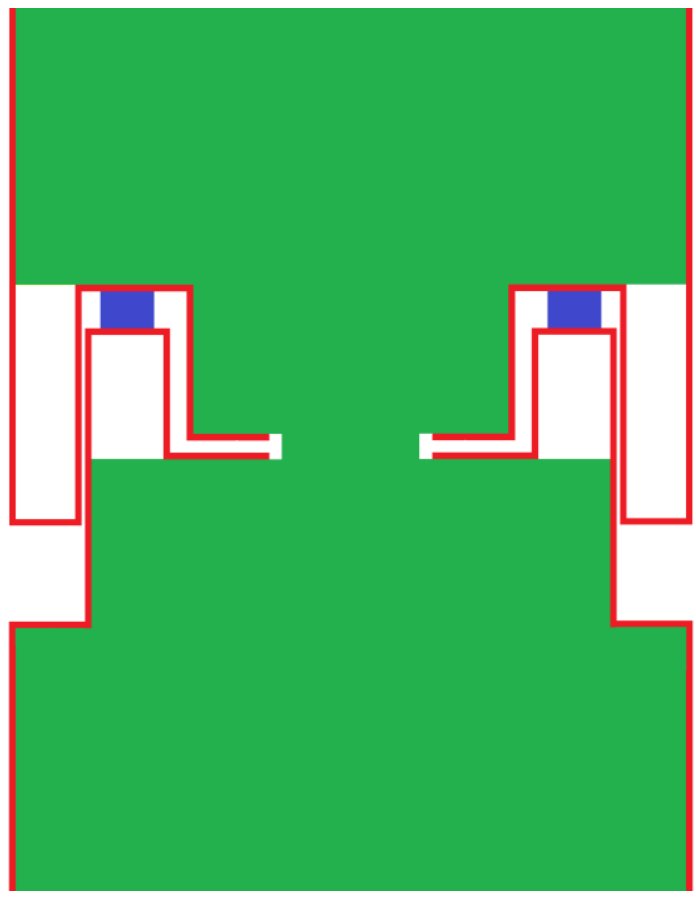

Fig. 1. Schematic picture of a panel's joint with sealants.
When calculating linear water vapour transmittance $\psi_{\mathrm{v}}[\mathrm{kg} / \mathrm{msPa}]$ full stationary water vapour flux $G_{0}[\mathrm{~kg} / \mathrm{s}]$ through the system and water vapour flux through the part without a water vapour bridge is needed. The second is zero in this study, thus $\psi_{\mathrm{v}}$ is calculated according to:

$$
\psi_{\mathrm{v}}=G_{0} / l\left(p_{\mathrm{v} 2}-p_{\mathrm{v} 1}\right),
$$

where $l$ is the length of the panel and $p_{\mathrm{v} 2}-p_{\mathrm{v} 2}$ is stationary vapour pressure difference between the inner and the outer part of the panel.

We have numerically examined panel joint with three different sealant combinations, which were experimentally investigated before [3]: joint without any sealant, with one EPDM sealant and with two EPDM sealants. For each of the panel joint (only) one experimental result with corresponding uncertainty is available. Our numerical models are two dimensional and consist of 4 steel parts, mineral wool and EPDM foam where built-in. Properties of used mineral wool in simulations are: density $\rho=120 \mathrm{~kg} / \mathrm{m}^{3}$ (taken from declaration), specific heat $c_{p}=1030 \mathrm{~J} / \mathrm{kgK}$ (taken from technical sheet), effective porosity $=0.9 \mathrm{~m}^{3} / \mathrm{m}^{3}$ (taken from Delphin 6.0), thermal conductivity $\lambda=0.040$ $\mathrm{W} / \mathrm{mK}$ and water vapour resistance factor $\mu=1.32$ (measured at ZAG). Sorption curve was taken from [4] for MW of type CNR. Water vapour resistance of the pure steel sheets of the thickness $0.7 \mathrm{~mm}$ is very high as discussed above; therefore, it was not measured for the sake of the study and was taken as infinity.

The dimensions of the researched "specimens" were similar as effective dimensions at experimental ones: height $=48 \mathrm{~cm}$ and thickness $=150 \mathrm{~mm}$ (experimental effective width of specimens was $48 \mathrm{~cm}$ ). The modelling in space was done with finite difference numerical scheme, modelling in time with CVODE or implicit Euler numerical scheme. Due to finite differences some approximation of the modelling geometry to rectangular geometry was done as it reads below. Heat and vapour transfer with condensation were calculated (heat transfer without radiation), whereas liquid moisture and air transport have not been taken into account. The order of magnitude of $G_{0}$ of systems under consideration is from $10^{-10}$ to $10^{-9} \mathrm{~kg} / \mathrm{s}$ and $\psi_{v}$ from $10^{-13}$ to $10^{-12} \mathrm{~kg} / \mathrm{msPa}$.

\section{Simulations and numerical results}

\subsection{Panel without a sealant}

Part of the model of the numerically researched panel without a sealant is presented in Fig. 2. This joint is without any sealant, thus there is only air between lips at the joint.

Due to different thermal conductivity the air is presented with different colours (white and black blue). This type of joints are not as much used on real objects, they were treated here mainly due to the research goal.

The model is a simplification of the real joint into rectangular geometry, whereas in that geometry the details of the original geometry, lengths and widths of air cavities, is captured as accurate as possible. The narrowest part of the joint, which plays crucial role in 
our study, is of the same length in the models as in the samples from experiments.

The experiment on that configuration lasted about 50 days. Estimated uncertainty in experimentally obtained $\psi_{v}$ is $0.9 \%$ (only) [3], whereby analytical model using for fitting experimental results was taken without uncertainty estimation.

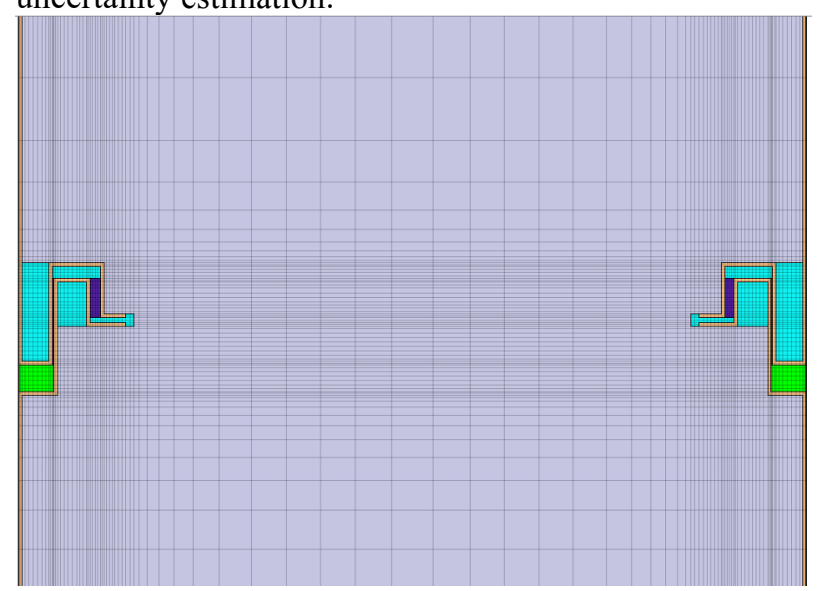

Fig. 2. Part of the numerical model with the joint without a sealant. Materials and numerical grid are shown Narrowest part of the joint $d_{G A P}=0.27 \mathrm{~mm}$ on both sides.

With Delphin 6.0 different configurations of the panels with different widths of the narrowest part of the gap $d_{G A P}$ were simulated (see Fig 3.). Boundary conditions are fixed and are the same as average experimental ones: $\mathrm{T}_{\text {wet }}=14.8{ }^{\circ} \mathrm{C}, \mathrm{T}_{\mathrm{dry}}=15.6^{\circ} \mathrm{C}, \mathrm{p}_{\mathrm{v} 1}=$ $1437 \mathrm{~Pa}$ and $\mathrm{p}_{\mathrm{v} 2}=18 \mathrm{~Pa}$.

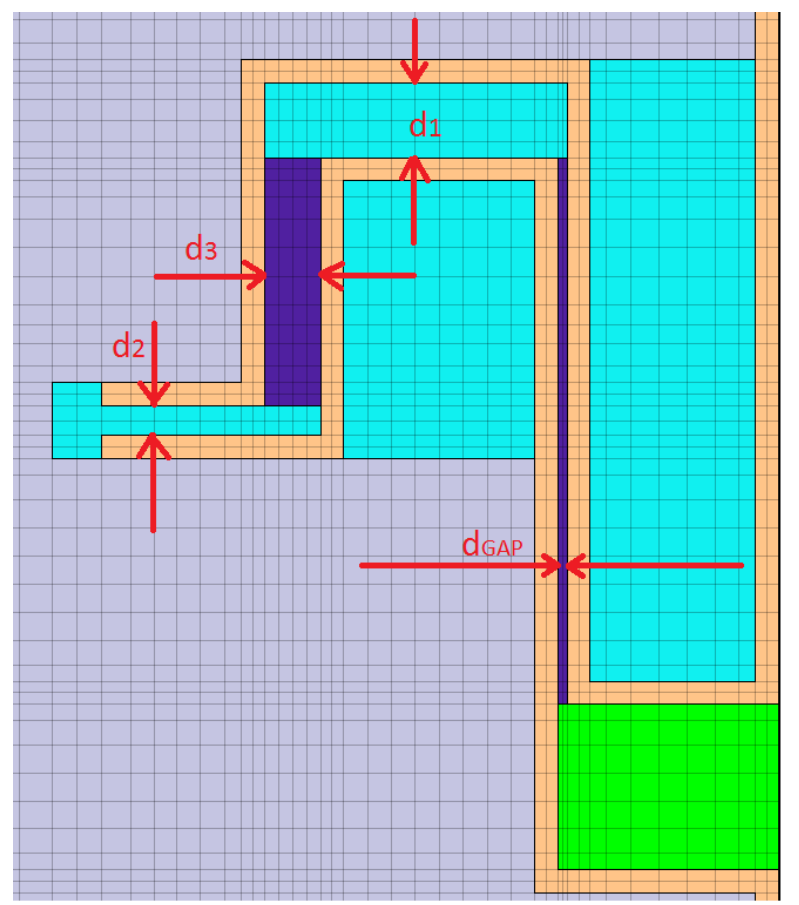

Fig. 3. Part of the numerical model of the joint without a sealant. Materials and numerical grid are shown. Narrowest part of the joint $d_{G A P}=0.27 \mathrm{~mm}$ on both sides.

Both narrowest gaps, at inner and at outer part of the panel are of the same width $d_{G A P}$ in numerical model. It was, of course, not the case at experimental specimens.
At the time of experimental work (2014) gap widths were not measured, unfortunately, later than the specimens were destroyed. It is actually not easy to measure $d_{G A P}$ at denser intervals through all the length of the joint except at the border of the panels. In reality one has a distribution of gap widths along each joint, but we operate here with mean values. It was estimated, that the gap widths vary between 0 and $1 \mathrm{~mm}$ from joint to joint. In second column of Table 1 relative differences between numerical and experimental results in $G_{0}$ (or $\psi_{v}$, relative values are the same) are written: $\psi_{v_{-} \text {num }} / \psi_{v_{-} \exp }-1$. Both values coincide when $d_{G A P}$ of both joints of width $0.27 \mathrm{~mm}$ is used in numerical simulation. Quite quick and large increasing of deviations from the "matching" configuration is seen if increasing or decreasing the $d_{G A P}$. It is to be emphasized that in numerical modelling both openings were always of the same width, whilst in experiment both openings are generally of different average widths, of course.

One has to state that results from numerical modelling are compared with only one experiment here, thus from the coinciding between experimental and numerical results none extrapolation to other panels can be done. Therefore, it is natural to look at the comparison between numerical results (with experimental vapour flux used) for any $d_{G A P}$ and those with mean gap width $d_{\text {GAP MEAN. For last we are taking }}$ $d_{\text {GAP_MEAN }}=0.5 \mathrm{~mm}$. The comparison is shown in third column of Table 1.

Table 1. Relative differences in linear water vapour transmittance in panel systems without a sealant when varying $d_{G A P}$. Matching configuration with the experiment is bolded.

\begin{tabular}{|c|c|c|}
\hline$d_{G A P}[\mathrm{~mm}]$ & $\begin{array}{c}\text { Relative difference } \\
\text { in } \psi_{v} \text { between } \\
\text { numerics and } \\
\text { experiment: } \\
\psi_{v_{-} \text {num }} / \psi_{v_{-} \text {exp }}-1 \\
{[\%]}\end{array}$ & $\begin{array}{c}\text { Relative difference } \\
\psi_{v} \text { between } \\
\text { numerics and } \\
\text { numerics at } d_{G A P}= \\
0.5 \mathrm{~mm}: \\
\psi_{v_{-} n u m} / \psi_{v_{-} n u m 0.5}-1 \\
{[\%]} \\
{[\%]}\end{array}$ \\
\hline 0.20 & -20 & -48 \\
\hline 0.27 & -0.1 & -35 \\
\hline 0.30 & +8.0 & -29 \\
\hline 0.40 & +32 & -13 \\
\hline 0.50 & +53 & 0 \\
\hline 1.00 & +123 & +46 \\
\hline
\end{tabular}

From Table 1 we see that for $d_{G A P}$ bigger than 0.2 $\mathrm{mm}$ it is possible to determine linear water vapour transmittance of any of our panels without a sealant to precision of about $\pm 50 \%$. For $d_{G A P}$ values below 0.2 $\mathrm{mm}$ (or even above $1 \mathrm{~mm}$, which are less probable) the deviations are larger. 
Changing the air layer widths $d_{1}, d_{2}$ and $d_{3}$ (see Fig. 3 ) is less influential to the vapour flux, because average values of $d_{1}, d_{2}$ and $d_{3}$ are larger than $d_{G A P}$ and relative change of $d_{1}, d_{2}$ and $d_{3}$ from a joint to another joint due to production and montage is smaller than the change of $d_{G A P}$. Therefore, a systematical study when changing $d_{l}$, $d_{2}$ and $d_{3}$ was not done here.

\subsection{Panel with a sealant}

The panel is of the same configuration as the panel without a sealant, except additional sealant at outer part of the joint. The sealant appears as the tube in compressed form made of EPDM. Due to tighter configuration than in the case without a sealant more time is needed here to reach the stationary state of water vapour transfer with constant boundary conditions. Therefore, the measurement (presented in [3]) was conducted for about 95 days. $\Psi_{v}$ in that experiment is for a factor of 3.12 lower than in experiment described under point 3.1 .

Material parameters for elastomeric foam (except for $\left.\mu_{E P D M}\right)$ used in simulations, were taken from Delphin 6.0. Boundary conditions are again the same as average experimental ones: $T_{\text {wet }}=20.4^{\circ} \mathrm{C}, T_{d r y}=21.7^{\circ} \mathrm{C}, p_{v l}=$ $2083 \mathrm{~Pa}$ and $p_{v 2}=104 \mathrm{~Pa}$. The estimated uncertainty in experimental $\Psi_{v}$ is the same as in experiment for the panel without a sealant, i.e. $0.9 \%$ [3].

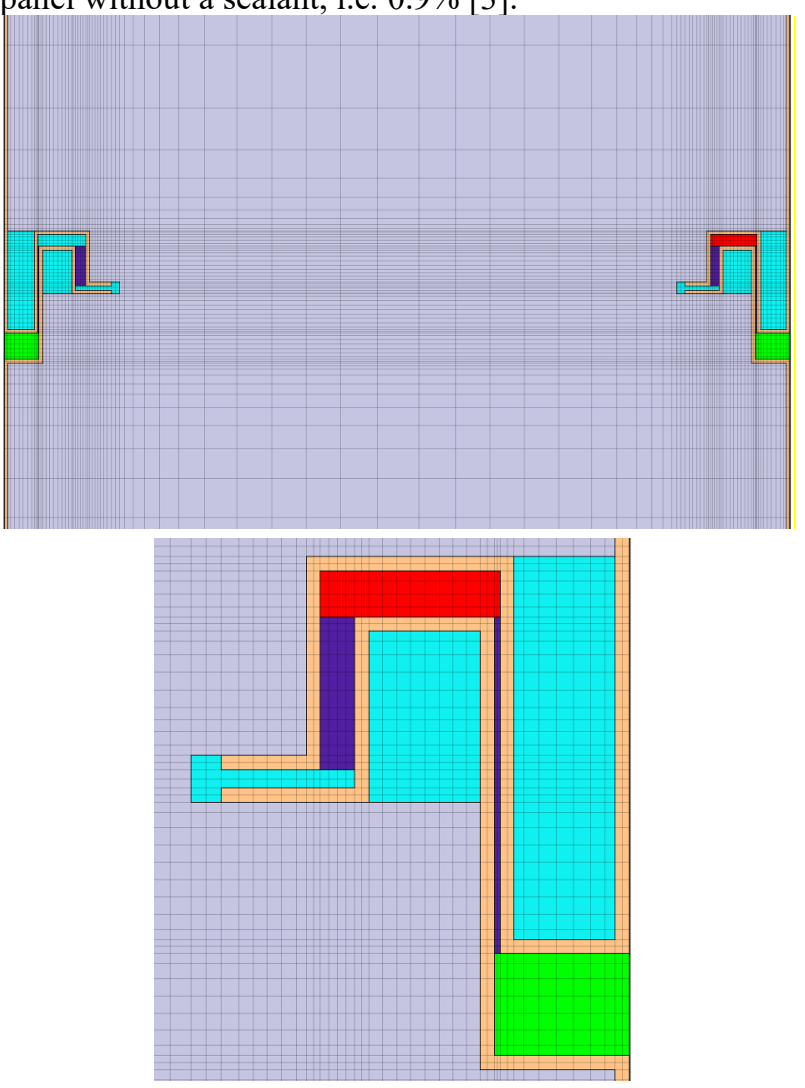

Fig. 4. (upper) part of a numerical model with joint with a longer rectangular sealant. Materials and numerical grid are shown, where sealant is in red colour. Narrowest parts of the joint $d_{G A P}=0.27 \mathrm{~mm}$ on both sides; (lower) upper picture enlarged.
When simulating systems with the sealant the parameters for all others except the sealant are the same as in simulating systems without it. For the starting $d_{G A P}$ the coinciding value $0.27 \mathrm{~mm}$ from the procedure described in 3.1 is taken into account, whereas the real value of $d_{G A P}$ in that experiment was not known indeed and could be in principle any value from 0 to $1 \mathrm{~mm}$. The sealant is in form of a compressed EPDM tube, whereas due to its shape in compressed state it is not possible to know its water vapour diffusion resistance factor using standardised procedures.

A Delphin 6.0 model with one possible configuration of the compressed sealant is presented in Fig. 4. The length of the sealant is $9 \mathrm{~mm}$ in this case and it fills all available place of horizontally oriented air cavity. In real joints this sealant is not fully horizontal oriented and not in the shape of rectangle, of course. But for purpose of this study, where 1.) numerical package with finite difference modelling working with rectangular geometry only is used and 2.) relative differences and not absolute values of sealant's water resistance are the pursued goal to here, the chosen approximated rectangular shape of the sealant is taken as suitable enough.

We found out, that (for the chosen shape of the sealant and air cavities in simulations) numerically and experimentally obtained results coincides, if at $d_{G A P}=$ $0.27 \mathrm{~mm} \mu_{E P D M}=35$ is set in simulations (Table 2).

Table 2. Relative differences in vapour fluxes through panel systems with a sealant when $d_{G A P}=0.27 \mathrm{~mm}$ and $\mu_{E P D M}$ is varying. Matching configuration is bolded.

\begin{tabular}{|c|c|}
\hline $\boldsymbol{\mu}_{\text {EPDM }}$ & $\begin{array}{c}\text { Relative difference in } \boldsymbol{G}_{\boldsymbol{0}} \\
\left(\text { or } \psi_{v}\right) \text { between numerics } \\
\text { and experiment [\%] }\end{array}$ \\
\hline 32 & +4.2 \\
\hline $\mathbf{3 5}$ & $\mathbf{- 0 . 5}$ \\
\hline 39 & -6.7 \\
\hline
\end{tabular}

As already written, in reality we do not know exactly the size of $d_{G A P}$. In order to study the sensitivity of $\mu_{E P D M}$ to $d_{G A P}$, we calculated $\mu_{E P D M}$ for chosen shape of the sealant (and joint) also for other values of $d_{G A P}$. With these $\mu_{E P D M}$ values (together with chosen $d_{G A P}$ values) the same stationary vapour flow is obtained in simulations as in the corresponding experiment. The results are shown in second column of Table 3. 
Table 3. Matching $\mu_{E P D M}$ values for the panel systems with a longer rectangular sealant with varying $d_{G A P}$.

\begin{tabular}{|c|c|c|}
\hline $\boldsymbol{d}_{G A P}[\mathrm{~mm}]$ & $\boldsymbol{\mu}_{E P D M}$ & $\begin{array}{c}\text { Relative difference } \\
\text { in } \boldsymbol{\mu}_{E P D M} \text { between } \\
\text { numerics and } \\
\text { numerics at } \boldsymbol{d}_{G A P}= \\
\mathbf{0 . 5} \mathbf{~ m m : ~ [ \% ] ~}\end{array}$ \\
\hline 0.20 & 26 & -43 \\
\hline 0.27 & 35 & -24 \\
\hline 0.5 & 46 & 0 \\
\hline 1.0 & 54 & +17 \\
\hline
\end{tabular}

If $d_{G A P}$ increases, also $\mu_{E P D M}$ must increase, because the same vapour flow is required. In third column of Table 3 the difference in matching $\mu_{E P D M}$ values between results with $d_{G A P}$ written in second column and result with the average $d_{G A P}=0.5 \mathrm{~mm}$ is shown.

From the research we see that, if narrowest gap width is above $0.2 \mathrm{~mm}$, it is possible to determine effective water vapour diffusion resistance factor of the sealant of the measured panel to precision of about $-45 \%$ to $+20 \%$. This would then be uncertainty in the quantity, if one was to install the panel in a real building and wanted to simulate it.

We simulated also panel with shorter (again rectangular) sealant of the length of $5.5 \mathrm{~mm}$ and the same width as sealant from Fig. 4. Part of this model is shown in Fig 5.

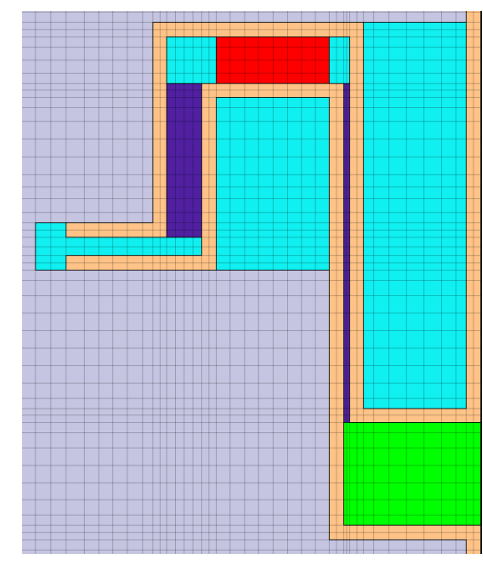

Fig. 5. Part of a numerical model with joint with a shorter rectangular sealant. Materials and numerical grid are shown, where sealant is in red colour. Narrowest parts of the joint $d_{G A P}$ $=0.27 \mathrm{~mm}$ on both sides.

Resulting $\mu_{E P D M}$ values for that model, at which numerically obtained vapour flows at a chosen $d_{G A P}$ coincide with corresponding experimentally obtained vapour flow, are shown in Table 4.
Table 4. Matching $\mu_{E P D M}$ values for the panel systems with a shorter rectangular sealant with varying $d_{G A P}$.

\begin{tabular}{|c|c|c|}
\hline$d_{G A P}[\mathrm{~mm}]$ & $\mu_{E P D M}$ & $\begin{array}{c}\text { Relative difference } \\
\text { in } \mu_{E P D M} \text { between } \\
\text { numerics and } \\
\text { numerics at } d_{G A P}= \\
0.5 \mathrm{~mm}:[\%]\end{array}$ \\
\hline 0.20 & 51 & -41 \\
\hline 0.27 & 68 & -21 \\
\hline 0.5 & 87 & 0 \\
\hline 1.0 & 99 & +14 \\
\hline
\end{tabular}

Final absolute $\mu_{E P D M}$ value depends much on the shape of the sealant, whereas we assumed that the width of the sealant is in both cases all available width of the air cavity. But relative difference of coinciding $\mu_{E P D M}$, when changing $d_{G A P}$, is very similar as that in Table 3 for longer sealant, even if vapour flow enters and leaves the sealant in horizontal direction in one case and in vertical direction in another case.

In Fig. 6 we see a model with an "elliptic" sealant, whereas in Table 5 the resulting $\mu_{E P D M}$ values for that model, at which numerically obtained vapour flows at a chosen $d_{G A P}$ coincide with corresponding experimentally obtained vapour flow.

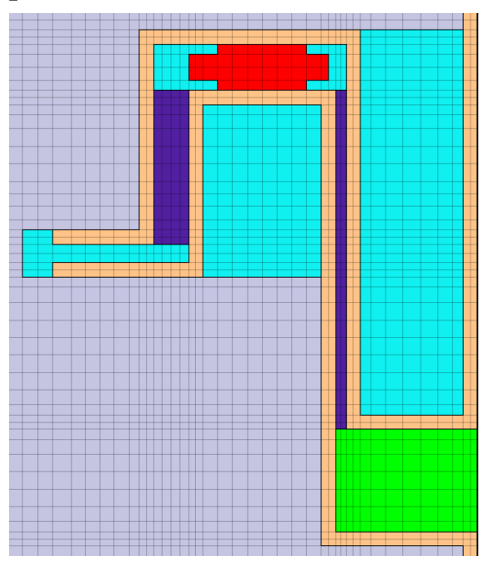

Fig. 6. Part of a numerical model with joint with an "elliptic" sealant. Materials and numerical grid are shown, where sealant is in red colour. Narrowest parts of the joint $d_{G A P}=0.5 \mathrm{~mm}$ on both sides. 
Table 5. Matching $\mu_{E P D M}$ values for the panel systems with an "elliptic" sealant with varying $d_{\text {GAP }}$.

\begin{tabular}{|c|c|c|}
\hline $\boldsymbol{d}_{G A P}[\mathrm{~mm}]$ & $\boldsymbol{\mu}_{E P D M}$ & $\begin{array}{c}\text { Relative difference } \\
\text { in } \boldsymbol{\mu}_{E P D M} \text { between } \\
\text { numerics and } \\
\text { numerics at } \boldsymbol{d}_{G A P}= \\
\mathbf{0 . 5} \mathbf{~ m m : ~ [ \% ]}\end{array}$ \\
\hline 0.20 & 73 & -32 \\
\hline 0.5 & 107 & 0 \\
\hline 1.0 & 128 & +20 \\
\hline
\end{tabular}

From comparison of Table 5 with Tables 3 and 4 one can conclude that the shape of the sealant influences beside absolute values also the relative differences and is thus important also in that way.

\subsection{Panel with two sealants}

Another measurement was done on the panel with two EPDM sealants: one sealant at the outer part and another at the inner part of the joint; it is also presented in [3]. $\Psi_{v}$ in that experiment is for a factor of 5.44 lower than in experiment under point 3.1. and for a factor of 1.74 lower than in experiment under point 3.2.

In numerical model both sealants are the short option from 3.2. Its model is shown in Fig. 7. Boundary conditions in numerical simulations equal the average experimental ones again: $T_{\text {wet }}=18.6^{\circ} \mathrm{C}, T_{d r y}=22.0^{\circ} \mathrm{C}$, $p_{v l}=1893 \mathrm{~Pa}$ and $p_{v 2}=80 \mathrm{~Pa}$. The estimated uncertainty in experimental $\psi_{v}$ is $4.5 \%$ [3]. All other material parameters are the same as at the point 3.2. For the $d_{G A P}$, the value $0.27 \mathrm{~mm}$, obtained according to the procedure described in 3.1 was taken into account for starting simulations. Consequently, for the $\mu_{E P D M}$ the matching value 68 from point 3.2 is taken into account.

In that case the numerically obtained resulting $\psi_{v}$ at panels with 2 sealants is for $38 \%$ higher than experimental ones.

We sought the coinciding $\mu_{E P D M}$ value in numerics also for that experiment. The results with relative differences are presented in Table 6 . For $d_{G A P}=0.5 \mathrm{~mm}$ the resulting $\mu_{E P D M}=112$ is for $28 \%$ above the corresponding value in described procedure applied on panel with only one sealant (Table 4).

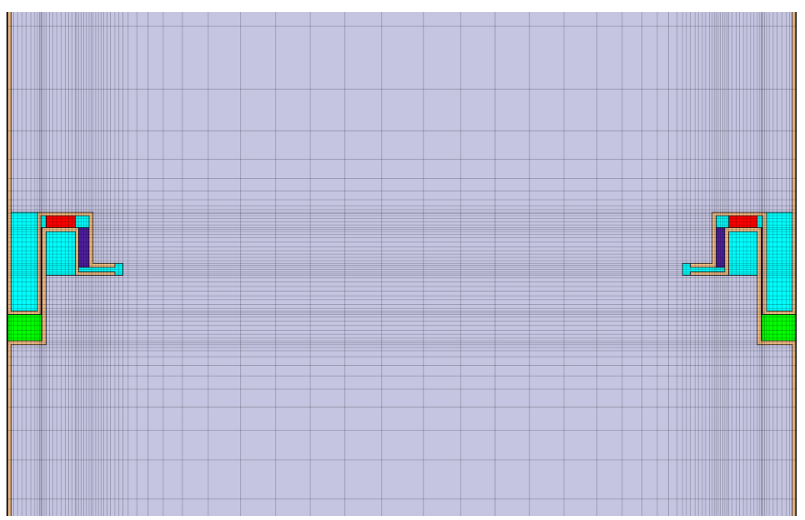

Fig. 7. Part of a numerical model with panel with two shorter rectangular sealants. Materials and numerical grid are shown, where sealants are in red colour. Narrowest parts of the joint $d_{G A P}=0.27 \mathrm{~mm}$ on both sides.

Relative differences for panels with two sealants roughly equals the relative differences obtained for panels with one sealant of equal shape divided by two. The most discrepancy from expected regime is at coinciding value at $d_{G A P}=1 \mathrm{~mm}$, where value about $7 \%$ was intuitively expected.

Table 5. Matching $\mu$ EPDM values for the panel systems with two shorter rectangular sealants with varying $d_{G A P}$.

\begin{tabular}{|c|c|c|}
\hline $\boldsymbol{d}_{G A P}[\mathrm{~mm}]$ & $\boldsymbol{\mu}_{E P D M}$ & $\begin{array}{c}\text { Relative difference } \\
\text { in } \boldsymbol{\mu}_{E P D M} \text { between } \\
\text { numerics and } \\
\text { numerics at } \boldsymbol{d}_{G A P}= \\
\mathbf{0 . 5} \mathbf{~ m m : ~ [ \% ]}\end{array}$ \\
\hline 0.20 & 91 & -19 \\
\hline 0.27 & 101 & -10 \\
\hline 0.5 & 112 & 0 \\
\hline 1.0 & 117 & +4.5 \\
\hline
\end{tabular}

\section{Discussion and Conclusions}

In the paper we numerically studied stationary water vapour transmission through sandwich panels. We had few laboratory experimental results from [3] available for comparison. Our goal was to find out to what precision it is possible to find water vapour resistance factor of a sealant in order to simulate the panel systems in real random climatic conditions without knowing the (distribution of) gap widths and parameters of the sealant in advance. Therefore, we were interested in studying of sensibility of linear water vapour transmittance to narrowest gap width at the joint of two panels and to the shape and water vapour resistance of compressed EPDM sealants.

With procedure proposed we determined the coinciding value for each configuration, i.e. the value at which numerical modelling and experiment give the same result and tried to do some general conclusions 
from the obtained results. Unfortunately, only one experiment was available for each configuration studied. Nevertheless, numerical results are meaningful and show quite big general sensitivity to the studied parameter narrowest gap width $d_{G A P}: \pm 50 \%$. In the case of effective water vapour resistance of the sealant the uncertainty for the measured panel with a sealant was about $-45 \%$ and $+15 \%$. It was about two times lower in the case of panel with two sealants. But determination of upper absolute effective water vapour resistance is actually impossible with the method, because we have only one experiment. In order to get it, more samples of the same panel configuration should be measured and numerically analysed to find the coinciding effective vapour parameter dependence on $d_{G A P}$ for each measurement. Then conclusions about gap width and water vapour resistance of the sealant mean values and standard deviations based on the statistical properties/distribution could be done.

Nevertheless, our study shows that using numerical simulations in combination with laboratory experiments on panels is the right way for roughly (!) estimation of the unknown effective water vapour resistance factor of a sealant of a "strange" shape, without even modelling its exact shape. But when further modelling it, the shape must remain the same as in previous modelling in order to take the obtained $\mu_{E P D M}$ value as reliable.

But in order to do precise simulation of water vapour transfer and condensation according to EN 15026 with real hourly climatic conditions on a random chosen panel from the production line, if having tolerances in narrowest gap width of $\pm 0.5 \mathrm{~mm}$ or more, knowing the exact $d_{G A P}$, shape and water vapour resistance factor is almost inevitable. It depends, of course, also on what is the goal of intended simulations according to EN 15026.

\section{References}

1. J. Bach et al, Stahlbau 83, 461 (2014)

2. M. Kuhnhenne et al, Stahlbau 87, 1072 (2018)

3. G. Vidmar, F. Knez, Bauphysik 37, 229 (2015)

4. M. Jiřičková, R. Černý, Construction and Building Materials 20, 425 (2006) 